\title{
R. Joussaume, L. Laporte, et C. Scarre (dir.), 2007, Origine et développement du mégalithisme de l'ouest de L'Europe
}

Christine Boujot

\section{CpenEdition}

Journals

Édition électronique

URL : http://journals.openedition.org/rao/475

DOI : $10.4000 /$ rao.475

ISBN : 978-2-7535-1607-6

ISSN : 1775-3732

Éditeur

Presses universitaires de Rennes

\section{Édition imprimée}

Date de publication : 30 décembre 2007

ISBN : 978-2-7535-0574-2

ISSN : 0767-709X

Référence électronique

Christine Boujot, «R. Joussaume, L. Laporte, et C. Scarre (dir.), 2007, Origine et développement du mégalithisme de l'ouest de L'Europe », Revue archéologique de l'Ouest [En ligne], 24 | 2007, mis en ligne le 24 janvier 2011, consulté le 04 décembre 2020. URL : http://journals.openedition.org/rao/475 ; DOI : https://doi.org/10.4000/rao.475 
Joussaume, R., Laporte, L. et Scarre, C. (dir.), 2007, Origine et développement du mégalithisme de l'ouest de L'Europe, actes du colloque international tenu à Bougon du 26 au 30 octobre 2002, Bougon, Conseil général des Deux-Sèvres/ Musée des Tumulus de Bougon, 2 vol., 830 p.

Le thème du mégalithisme est un tellement vieux sujet que d'aucuns pourraient le croire épuisé. D’ailleurs les dernières préconisations en matière de programmation de la recherche archéologique pour les périodes néolithiques de la région de l'Ouest pointent sa surexploitation au détriment des données tirées des habitations et de l'économie.

Pourtant, le nombre d'ouvrages publiés récemment sur ce thème démontre au contraire sa grande vitalité. De plus, la mise en évidence d'une monumentalité funéraire " prémégalithique » en Bretagne, contemporaine de l'apparition de la monumentalité funéraire ailleurs en Europe, telle qu'elle fut actée par exemple au $17^{\mathrm{e}}$ Colloque interrégional sur le Néolithique à Vannes (1990) et dans sa publication de 1992, a constitué un apport suffisamment majeur de ces dernières années pour devenir un enjeu dans la compréhension de la néolithisation en France occidentale. Depuis lors, de nombreuses manifestations scientifiques se sont succédées pour débattre différents aspects liés au mégalithisme, dont la question de ses origines autour de laquelle fût spécifiquement construit le Colloque international de l'UISPP à Santiago de Compostela, en avril 1996 (publié en 1997) et le programme des séminaires du professeur Jean Guilaine au Collège de France durant l'année 1997.

Bien sûr, les perspectives ouvertes par cette piste de recherche sont loin d'être toutes explorées. Pour autant, proposer une nouvelle rencontre internationale sur ce thème laissait supposer un abord original, différent des précédents. Or, rien dans le titre ne révèle les choix ni même les objectifs retenus. Celui-ci affiche que le débat porte sur l'« Origine et le développement du mégalithisme de l'Ouest de L'Europe " comme s'il s'agissait d'un processus unique et infini.

En préambule à l'ouverture de ce colloque, les intentions sont toutefois dévoilées de réunir une synthèse des connaissances du mégalithisme (monuments funéraires, pierres dressées et l'art qui s'y rattache) pour l'ouest de l'Europe, en insistant sur les avancées les plus récentes. Cinq ans après la tenue de cette manifestation, les intentions sont tenues et le résultat est enfin matérialisé sous la forme d'une publication

l'objet que de deux publications provisoires (non mentionnées en bibliographie car sans doute parues après remise du manuscrit) : MÉnEZ, Y. et Gomez de Soto, J., 2006, L'habitat de Kergariou à Quimper, Bulletin de l'Association française pour l'Étude de l'Âge du Fer, 24, p. 83-85; MÉnEz, Y. et Gomez DE Soto, J., 2006, L'habitat de Kergariou à Quimper, Journée "Civilisations atlantiques et Archéosciences ", Rennes, 8 avril 2006 (résumés des communications), p. 28-29. éditée par le Conseil général des Deux-Sèvres : Musée des Tumulus de Bougon.

L'ouvrage est constitué de deux volumes. Le premier, qui regroupe plus de la moitié des contributions, est consacré aux monuments funéraires alors que le deuxième est réservé aux pierres dressées et à l'art mégalithique. Ainsi, les organisateurs de ce colloque nous proposent une vision du mégalithisme fondée sur deux grands ensembles, les « architectures funéraires » et les "pierres dressées ». Le choix de cette présentation matériellement distincte, probablement adopté par souci de commodité, ne va pas sans susciter quelques réticences dans la mesure ou il rompt toute relation entre ces différents aspects architecturaux du phénomène, au moment où leurs liens commencent et se préciser.

\section{Premier volume :}

\section{«Monuments funéraires (monde des morts) »}

Le premier volume est consacré aux monuments funéraires répertoriés dans un espace géographique désigné comme l'ouest de l'Europe et subdivisé en quatre grandes parties :

- le Nord de l'Europe, qui s'étend de la Scandinavie à la Belgique en traversant la plaine nord-européenne (AllemagnePologne); un détour s'impose par le Luxembourg afin de considérer, avec F. Valloteau, le premier menhir découvert sur ce territoire;

- la Grande-Bretagne et l'Irlande, jusqu'aux confins de l'Écosse et sans oublier l'île de Guernesey;

- la France, subdivisée à son tour en quatre grandes provinces traversées depuis le Centre-Sud (Massif central, Quercy, Aquitaine, Pyrénées centrales), la Méditerranée nord-occidentale (îles de la mer tyrrhénienne, Provence, Languedoc méditerranéen, Roussillon, Catalogne), les régions de l'Ouest (Normandie, Bretagne, Pays de Loire, Poitou), pour terminer par le Bassin parisien en poussant jusqu'en Suisse;

- la péninsule Ibérique, parcourue depuis les régions de l'intérieur en direction de l'Andalousie pour rejoindre ensuite le centre et le sud du Portugal.

Chacun des articles proposés explore non seulement ces vestiges architecturaux dans divers contextes géographiques mais également dans des contextes culturels et probablement sociaux tout aussi différents, étant donné que la période considérée couvre plusieurs millénaires (depuis le $v^{\mathrm{e}}$ jus-

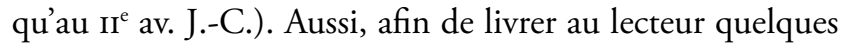
repères indispensables à son parcours de cet espace/temps 
considérable, une courte introduction précède chaque partie afin de placer le propos dans son contexte historique et géographique. Dans le même esprit, une traduction complète en anglais de chaque contribution est, de ce point de vue, particulièrement appréciable.

L'ensemble de l'ouvrage s'appuie de manière équilibrée sur d'importantes synthèses régionales, micro-régionales et interrégionales alternant avec des études de cas portant sur un thème particulier, ou présentant les résultats de sondages ou de fouilles d'un monument. Pour chacune de ces grandes aires géographiques, les monuments sont interrogés, observés, considérés selon différentes perspectives : depuis le choix de leur emplacement, des matériaux et des techniques, la conception et l'organisation des structures, les opérations de construction, les interventions d'entretien, de réfection, de remaniements, les modes d'utilisation et de fonctionnement, les opérations de fermeture, de condamnation et de destruction, la diversité typologique, les transformations affectant les édifices et les lieux au fil du temps. Enfin, le renouvellement des problématiques amène également à aborder ces architectures dans leur rapport à l'environnement, à la structure et à l'organisation de l'espace de vie, du territoire habité.

\section{Projets, conceptions, constructions et organisations des structures}

Dans la synthèse qu'ils nous présentent des monuments scandinaves, T. Dehn et S. I. Hansen traquent et pistent toutes les traces relatives aux procédés et techniques de construction des tombes mégalithiques pour nous livrer une série d'observations nouvelles et parfois inattendues. Cette quête ouvre sur un domaine encore peu exploité touchant au projet architectural lui-même, à sa conception, sa projection au sol par le tracé de "lignes de couloir".

L'exemple de la fouille du dolmen des Aguals (Gréalou-etMontbrun, Lot), rapporté par J.-P. Lagasquie, D. Barreau et A. Rocher livre également des observations relevant de cette étape du " projet » qui permettent de penser que l'ensemble des structures du monument a pu être conçu dans la phase initiale d'utilisation du dolmen.

\section{Le choix des matériaux}

Cet aspect spécifique fait l'objet de trois exposés sur la provenance géographique et géologique des blocs utilisés. Si les premiers résultats de l'étude menée en Belgique (S. Pirson, M. Toussaint, C. Frébutte) montrent une acquisition des blocs à proximité des lieux d'érection des monuments, ceux de l'analyse menée dans le département français du Puy-deDôme (A. de Goër de Herve et F. Sumerly) aboutissent à des conclusions opposées et démontrent, au contraire, un prélèvement des pierres éloigné du lieu d'implantation qui n'apparaît pas toujours nécessairement déterminé par la disponibilité locale en matériaux exploitables. Une approche comparable menée en Charente-Maritime par G. R. Colmont débouche sur des considérations relatives au transport des matériaux, aux distances parcourues et aux trajets empruntés.

La mise en évidence de l'emploi de pierres jumelles (deux parties issues d'un même bloc), disposées côte à côte ou l'une en face de l'autre dans près de la moitié des tombes scandinaves, apporte à cette question un éclairage tout à fait singulier (T. Dehn et S. I. Hansen).

\section{Les opérations de construction}

Nombre de recherches s'attachent à détecter, identifier et analyser les techniques utilisées dans la construction de tombes mégalithiques. Les exemples scandinaves, avec la présence de traces d'écorces de Bouleau, de boue crayeuse et d'argile qui ont été décelées entre chaque assise de pierres pour assurer l'étanchéité des chambres, apportent les informations les plus nouvelles dans ce domaine (T. Dehn et S. I. Hansen). Enfin, les traces de défauts, d'erreurs, d'accidents produits au moment de la construction et les réparations effectuées par les bâtisseurs eux-mêmes sont repérées et observées sur des monuments Danois.

La présentation du type particulier des dolmens à murs latéraux en pierre sèche de Languedoc oriental permet à M. Bordreuil, M.-C. Bordreuil et L. Jallot d'aborder différemment cet aspect des techniques de construction en soulevant la question du remplacement d'un procédé par un autre, du remplacement de l'emploi de dalles mégalithiques par de la pierre sèche.

\section{Des architectures diverses}

Tout au long de cette traversée des différents espaces d'Europe de l'ouest, chaque étape est l'occasion de présenter et/ ou de rappeler au lecteur les grandes catégories ou principaux types de monuments classiquement reconnus : les longs tumulus avec ou sans chambre, les cistes, les sépultures sous dalles, les dolmens (à cour, à portique, à couloir, cunéiformes, portal dolmens, tholö̈, passage graves, clava cairns, allées couvertes et allées sépulcrales, etc.). Chacune de ces catégories comprend tout un panel d'édifices dont les types tendent à s'affiner, à se préciser et à se multiplier au fur et à mesure des acquis de la recherche qui révèlent chaque année des structures élaborées selon de nouvelles combinaisons de matières, intégrant parfois des paramètres comme la couleur et la texture.

Cet inventaire confirme qu'en dehors des architectures colossales bâties de grosses dalles, le mégalithisme recouvre aussi de nombreux édifices parfois beaucoup plus discrets et composés de matériaux plus « légers ». Pour cette raison, la plupart des auteurs posent la question de savoir ce que 
l'on entend au juste par le terme "mégalithique ». Et c'est pourquoi J. N. Graham Ritchie, par exemple, annonce qu'il entend traiter des édifices mégalithiques écossais en les inscrivant dans une conception beaucoup plus large de la monumentalité architecturale (funéraire ou non), incluant les réalisations architecturales en bois.

Cette grande diversité de types de monuments revient sous presque toutes les plumes. Soulignée dans chaque synthèse, elle y est souvent mise en correspondance avec des variations régionales et/ou culturelles comme, par exemple, dans l'exposé proposé par M. S. Midgley sur les tombes mégalithiques de la plaine nord-européenne. Toutefois, d'autres manières d'appréhender cette variabilité sont discutées, notamment avec C. Scarre, R. Joussaume et L. Laporte qui la perçoivent également comme le résultat de l'histoire complexe des édifices, avec ses différentes phases de construction, de réfection, de condamnation et de destruction.

Par ailleurs, C. Scarre souligne la richesse que l'on est en droit d'attendre, sur ce sujet, d'une démarche comparatiste. En effet, au-delà des différences, l'importance des parallèles entre les monuments du sud de la Grande-Bretagne avec ceux de régions voisines (Irlande, Écosse, pays de Galles et Armorique), lui apparaît significative de contacts et d'échanges. Pour autant, peu de contributions adoptent ce type d'approche à l'exception de quelques rares tentatives parmi lesquelles la synthèse interrégionale présentée par J.-P. Daugas pour le centre-sud de la France, par exemple, qui vise essentiellement à identifier des groupes architecturaux spécifiques pour délimiter ensuite leurs sphères d'influences au-delà des frontières régionales.

Au regard de quelques nouvelles découvertes comme, par exemple, la reconnaissance partielle d'un cairn observé récemment par sondages sous le château d'Angers, cairn qui laisse deviner des traits typologiques analogues à ceux d'autres régions aussi éloignées que le sud-ouest de l'Armorique (C. Marcigny, E. Ghesquière, C. Hugot), on peut regretter que ces démarches comparatistes ne soient pas, ici, plus développées.

C'est encore une fois la distribution spatiale des tombes irlandaises qui permet d'y distinguer trois grands groupes régionaux. Toutefois, prenant acte que ces trois grandes catégories diffèrent également par leur contenu mobilier, G. Eogan tente leur mise en perspective chronologique, multipliant ainsi les procédures d'interprétation de cette diversité.

\section{Chronologie et question des origines}

Toutes les contributions se consacrent à cet essai de périodisation. Mais, étant donné la disparité des manifestations architecturales souvent utilisées sur une très longue durée, l'exercice s'en retrouve parfois nécessairement limité. Par un exposé ancré dans une rétrospective historiographique,
J. Guilaine se place délibérément dans cette longue durée pour nous proposer d'observer l'évolution architecturale du "mégalithisme dolménique " de méditerranée nordoccidentale au fil de plusieurs millénaires ( $\mathrm{v}^{\mathrm{e}}-\mathrm{II}^{\mathrm{e}}$ av. J.-C.), depuis leur genèse (proto-mégalithisme) jusqu’à leur déclin, phase ultime cette fois intégrée. Cette observation, menée par grandes aires culturelles, prend en considération l'évolution dans la façon d'utiliser les monuments.

À l'intérieur de l'espace géographique compris depuis le nord de l'Europe jusqu'aux régions du sud de la France, les monuments contenant des caveaux fermés sont placés à l'origine du "phénomène ». Apparaissant dans la première moitié $\mathrm{du} \mathrm{V}^{\mathrm{e}}$ millénaire, ils se présentent sous la forme de longs tumulus de terre dans la plaine nord-européenne (M. S. Midgley), de tumulus gigantesques ou encore de tertres plus discrets, allongés ou circulaires dans les régions de l'Ouest (R. Joussaume et L. Laporte), de longs tumulus dans le centre du Bassin parisien (J. Leclerc et J. Tarrête), de caissons de pierre logés dans des tumulus allongés ou circulaires en méditerranée nord-occidentale (J. Guilaine), dont la Corse (F. de Lanfranchi).

Outre les approches de synthèses, plusieurs enquêtes de terrain sont conviées également pour apporter leur écot à ce dossier des origines. Qu'il s'agisse de l'exemple de la nécropole de La Jardelle à Dissay (Vienne), rapporté par J.-P. Pautreau, Bernard Farago-Szekeres et P. Mornais, ou encore ceux du Monte Revincu ou de Mamucci dans la région du Nebbio/Agriate (Haute-Corse) présentés par F. Léandri, C. Gilabert et F. Demouche, la plupart de ces exemples corroborent les hypothèses précédentes tout en leur apportant quelques précisions.

Au cœur de la péninsule Ibérique, la situation semble un peu différente selon P. Bueno Ramirez, R. de Balbin Behrmann et R. Barroso Bermejo; ils s'arrêtent sur la difficulté rencontrée pour y établir une primauté entre coffres et tombes à couloir dans la genèse du phénomène mégalithique. Ce problème est également souligné par R. Joussaume et L. Laporte qui adoptent la position selon laquelle, pour l'ouest de la France, certaines tombes à couloir à chambres circulaires et/ou quadrangulaires partagent avec les caveaux fermés les premières manifestations de la monumentalité funéraire.

Quoi qu'il en soit, il en ressort surtout que ce sont également sur ces tout premiers monuments que semblent fondées les premières matérialisations de limites, de bornes, de marqueurs territoriaux.

\section{Position géographique, impact dans le paysage et relations spatiales}

En raison de leur impact visuel, ces architectures sont interrogées dans leurs différentes relations spatiales avec leur environnement. 


\section{Rapports entre architectures et environnement}

Le premier lien fondamental, établi par l'ensemble des chercheurs, concerne celui qui relie tout d'abord les différentes formes de monuments. Le travail mené par Y. Pailler et $Y$. Sparfel, qui tisse tout un réseau de relations sur l'ensemble mégalithique de Zoulierou (île Molène, Finistère), est à cet égard exemplaire.

Autour des grandes allées couvertes de la région de Wéris en Belgique, c'est également le champ des relations avec les autres sépulcres (notamment en abris naturels), les pierres dressées puis, également, avec les indices d'habitats que se proposent d'explorer M. Toussaint, C. Frébutte, S. Pirson, F. Hubert et P. Massy. Dans la région neuchâteloise, M.-H. Grau Bitterli et A. Leducq nous invitent à les suivre sur le plateau de Bevaix où les aménagements mégalithiques peuvent être considérés dans un espace constitué de structures de combustion, de fosses, d'aires à vocation artisanale etc.

J. N. Graham Ritchie s'attache également à saisir l'organisation de l'espace des îles Orcades en esquissant des relations entre l'architecture des tombes, des henges et des habitations. S'intéressant à l'orientation spatiale de celles-ci, il nous conduit bien au-delà du seul champ terrestre, dans une dimension intégrant les rapports avec les mouvements célestes et l'astronomie. Peu exploité, ce type de problématique est, par ailleurs, abordé par R. Oldham sur la base de la mesure précise des orientations des couloirs des tombes de la région portugaise de l'Alentejo.

Dans ce domaine des relations spatiales, beaucoup d'autres travaux sont présentés qui rendent compte de la diversité des liens entre les édifices mégalithiques et les autres repères artificiels et naturels de l'environnement. Ainsi, le regard porté sur les monuments de la province de Séville par L. Garcia Sanjuan et D. W. Wheatley, dans le cadre d'une étude menée conjointement par les universités de Séville et de Southampton, procède d'une vision tout à fait nouvelle, qui scrute et tente d'analyser les changements de comportements des populations qui, au fil du temps, ont voisiné avec ce repère "mégalithique » stable et permanent.

Sur ce sujet des interactions monumentalités/environnements, le lecteur trouvera, au gré de sa lecture, beaucoup d'autres témoignages susceptibles de le guider, de l'accompagner dans son parcours de tous les espaces-clés que sont les monts, les vallées, les rivières, les gués, les îles, le ciel etc., afin d'observer ici et là les règles d'implantation des mégalithes.

Au fil des contributions et de ces expéditions aux quatre coins de l'Europe occidentale, de nombreuses questions sont abordées avec différents auteurs. Pour autant, on peut regretter que, depuis le choix de l'emplacement et des matériaux, via les différentes étapes de construction et jusqu'à l'ins- cription de l'ouvrage définitif dans le paysage, ces analyses n'intègrent pas les rapports du dehors au dedans, ou encore de l'intérieur du tombeau à l'extérieur, des rapports entre le monde des morts et celui des vivants.

De la même manière, les interprétations sociales sont à peine esquissées à l'exception de quelques rares contributions, dont celle de G. Eogan qui nous place de plain-pied face à cette problématique. En effet, les monumentaux dolmens à cour localisés dans la région la plus fertile d'Irlande, où de véritables paysages agraires néolithiques ont été découverts sous les tourbières, nous sont présentés comme des productions significatives de l'existence de véritables "fortunes» au sein des premières sociétés d'agriculteurs. Dans un registre similaire, l'implantation des mégalithes par rapport à l'environnement peut être également mise en corrélation avec l'ouverture du milieu, l'anthropisation de la "Garrigue » au Néolithique final (J. Guilaine).

Quelques propositions sont ainsi esquissées qui voient dans l'émergence de la monumentalité funéraire l'expression de nouvelles représentations de l'espace liées à la sédentarisation des hommes et aux changements intervenus dans leurs pratiques culturales. Pour la plaine nord-européenne, M. Midgley avance même la notion de « création de paysage cérémoniel »! Sans aller jusque-là, il apparaît sans ambiguïté que les architectures mégalithiques tiennent une place conséquente dans la structuration, la construction de l'espace de vie, du territoire habité.

\section{Utilisations et pratiques funéraires}

Alors précisément, cet espace que l'on découvre, on s'attendrait un peu à le voir utilisé. Or, rien ne perce (ou pas grand-chose) de cette dimension essentielle, pourtant mise en exergue ces dernières années par de nombreuses études de sépultures qui ont montré et démontré tout l'intérêt de la recherche des gestes funéraires pour la compréhension non seulement des pratiques mortuaires mais également des architectures.

Le travail conduit par J. Leclerc et J. Tarrête figure parmi ceux qui se réfèrent à ces dernières approches pour retenir l'attention sur le développement autonome de trois phénomènes que sont la monumentalité, le mégalithisme, l'inhumation collective et la manière dont ils vont se rencontrer dans une région comme le Bassin parisien.

Dans la perspective qu'il nous soumet de l'évolution architecturale des dolmens de méditerranée nord-occidentale, J. Guilaine prend également en considération les informations fournies par les études ostéo-archéologiques afin de tracer les grandes lignes d'une trajectoire, d'une évolution dans la façon d'utiliser ces architectures.

En dehors de la synthèse de P. Aguayo de Hoyos et de L. Garcia Sanjuan, qui s’ajoute aux précédentes pour présen- 
ter le mégalithisme andalou en associant la morphologie des architectures et les pratiques funéraires, la grande majorité des communications ne retient pas cette option.

Certes, la connaissance des pratiques funéraires nécessite de disposer d'une documentation suffisante. Or, dans la plupart des cas, si les vestiges d'architectures permettent encore d'avancer dans ce dédale de couloirs et de chambres, des hommes ne restent même plus les os. Et si, par chance, les données conservées permettent d'aborder cet aspect, c'est l'infinie variété des modes d'utilisation et de réutilisation des sépulcres qui est soulignée, simplement énumérée, sans jamais tenter d'associer le corps (mort mais aussi vivant) et l'espace.

Le lecteur ne sera pourtant pas à l'abri de quelques surprises en découvrant, par exemple, le contenu intact d'une petite cellule dérobée derrière le mur de la chambre principale d'un dolmen des îles Orcades. En Eure-et-Loir, C. Verjux lui fera la démonstration du fonctionnement d'un type particulier de tombe nommé "sépulture sous dalle ", mettant en scène cette dalle comme dispositif de condamnation plutôt que de couverture.

\section{Politiques de protection, de restauration, de mise en valeur et d'aménagements}

Cet ensemble de contributions livre à la connaissance de tous la richesse des informations qu'il est possible d'obtenir à partir des études de ces vestiges. Ces contributions nous amènent, dans bien des cas, à prendre la mesure des transformations qui ont affecté les architectures et les lieux au fil du temps et de l'urgence d'en conserver la mémoire et les vestiges subsistants. Le Danemark, par exemple, compte 2500 tombes mégalithiques classées, et on pense que cet effectif représente $10 \%$ du nombre de tombes ayant existé. Aussi, c'est avec J.-P. Daugas, L. Devillard et B. Gely que nous allons refermer ce volume sur la question cruciale des politiques et mesures de protection et de conservation qui ne suffisent plus à protéger ce patrimoine menacé de disparaître.

\section{Deuxième volume : pierres dressées, art mégalithique (monde des vivants?)}

L'objet affiché du premier volume étant les «monuments funéraires ", tout laisse penser que le deuxième volume, consacré aux pierres dressées et à l'art mégalithique, s'ouvre alors sur le monde des vivants.

\section{Les Pierres dressées}

On aborde ce deuxième grand aspect du mégalithisme en empruntant un cheminement quelque peu différent de celui déjà effectué dans la reconnaissance des édifices sépulcraux : depuis la Grande-Bretagne et l'Irlande pour accoster dans l'ouest de la France avant de gravir les montagnes de l'aire alpine et d'en dévaler les pentes jusqu'à la mer Méditerranée puis les confins de la Péninsule Ibérique. Tout comme le précédent, ce volume est construit sur une alternance bien proportionnée de synthèses et d'études de cas qui examinent ces vestiges dans des contextes culturels différents renvoyant à un même temps long (toujours entre les $\mathrm{V}^{\mathrm{e}}$ et $\mathrm{II}^{\mathrm{e}}$ millénaires).

On entre dans ce domaine des pierres dressées guidé par C.-T. Le Roux qui nous expose les difficultés à entreprendre leur étude. En effet, non seulement ces pierres ont pu être dressées à toutes périodes mais, communément accessibles, elles font l'objet d'une telle diversité de pratiques et de représentations que, finalement, le sujet a peu suscité de travaux. Pour l'ouest de la France, il en résulte que les recherches sur le mégalithisme ont surtout bénéficié aux assemblages de type "dolmens ». Ce constat justifie peut-être le choix d'avoir centré ce volume sur cet aspect moins connu du phénomène. Il explique peut-être aussi que les pierres dressées sont examinées ici essentiellement du point de vue des méthodes déployées pour les cerner, les identifier au plus près. En ce sens, c'est une opération de construction et de mise au point d'une procédure de classification des alignements de menhirs de la zone d'Avrillé-Le Bernard (Vendée) que propose G. Bénéteau-Douillard.

Bien que séparés des tumulus et dolmens, c'est pourtant souvent en référence à ces derniers que les édifices de pierres dressées commencent à pouvoir être saisis, perçus et parfois même localisés. L'importance du lien qui peut les unir a été déjà souligné dans plusieurs analyses du premier volume où l'inscription des monuments funéraires dans le paysage introduisait les notions de bornes, de limites, de territoires, de repère géographique. Notion dont il ressort qu'elle est la ligne de force de ce deuxième volume : tout comme les monuments funéraires, les pierres dressées interviennent dans la configuration géographique des lieux et permettent, en association ou non avec les autres édifices, de structurer l'espace. Cet impact dans le paysage, souligné par C.-T. Le Roux, est le dénominateur commun de toutes les contributions qui nous présentent différentes facettes d'approches spatiales.

L'analyse de R. Bradley insiste sur la singularité des ouvrages mégalithiques de Grande-Bretagne et d'Irlande, mettant l'accent sur l'extrême complexité de réseaux de sites au sein desquels les pierres dressées balisent non seulement les territoires mais jalonnent également des parcours.

Après un recensement des différentes manifestations du phénomène "pierres dressées " de toute l'aire alpine et de ses marges, A. D'Anna et L. Pinet nous entraînent pour une excursion à travers le versant oriental et méridional 
du Massif central, le Languedoc, la Provence, la Lunigiana et la Sardaigne pour s'attarder un peu plus longuement en Corse du sud sur le plateau de Cauria. Ce plateau, ils l'arpentent dans les moindres recoins dans une approche très complète du territoire associant l'archéologie, la géologie, la géomorphologie. Les sites d'alignements y apparaissent ainsi intégrés à des systèmes et réseaux d'établissements dès le $\mathrm{V}^{\mathrm{e}}$ millénaire.

M. Calado s'attache, quant à lui, à dégager une analyse de la distribution spatiale des menhirs de la péninsule Ibérique. Ils se répartissent en cinq grandes régions (Algarve, Alentejo, Centre-nord du Portugal et Galice, Corniche cantabrique et Pyrénées occidentales, Catalogne), pour chacune desquelles correspond un ensemble mégalithique spécifique à tel point qu'il est difficile d'envisager que tous procèdent de la même trajectoire historique, l'une d'elles (celle du sud-ouest) puisant probablement son origine dès le $\mathrm{V}^{\mathrm{e}}$ millénaire (voire même avant).

À travers le recours au SIG (Système d'Informations Géographique), ce sont les différentes dynamiques d'implantation des mégalithes de l'aire Tage-Sever que tente d'examiner E. Lopez Romero Gonzalez de la Aleja. Les tout premiers résultats présentés ici nous font miroiter les potentialités d'une telle approche. La fouille de sauvetage des menhirs des terriers (Les Arcs-sur-Argens, Var), conduite par P. Hameau, nous montre une autre facette des méthodes d'approche de ce type de site. Puis J.-C. Roudil et C. Tardieu, avec la découverte de la stèle du Bois-Noir à Banne (Ardèche) mettent en évidence une pierre travaillée et dressée à la manière d'une stèle anthropomorphe, pierre qu'ils ajoutent à la construction de cet ouvrage.

\section{L'art mégalithique}

Enfin, une troisième partie sur "l'art mégalithique " conclut l'ouvrage sur une synthèse des traditions artisti- ques liées aux mégalithes d'Irlande par M. O'Sullivan, un exposé critique de J.-L. Le Quellec concernant les " récents » développements sur l'art mégalithique en France et un essai méthodologique pour l'étude des techniques des gravures néolithiques armoricaines présenté par E. Mens.

Encore une fois, et notamment concernant ce dernier chapitre, on peut regretter le délai de parution de ces actes, délai que l'on comprend parfaitement lié à l'ampleur de la tâche mais qui fait que certaines informations datent déjà. Enfin, une dernière remarque concerne les sources bibliographiques de l'ensemble des contributions qui sont rassemblées en fin d'ouvrage, probablement par souci de ne pas trop alourdir la présentation. C'est un parti que l'on peut regretter lorsque l'on sait la part importante de l'enquête bibliographique dans la démarche personnelle de chaque chercheur. Cela signifie aussi, hélas, que chacun ne peut reproduire sa contribution sous forme de tiré à part. Ceci étant dit, ces dernières réserves ne sauraient occulter les qualités de l'ouvrage, qui a pour autre atout une traduction intégrale des textes en anglais par C. Scarre.

Cet ouvrage présente à plus d'un égard une grande utilité pour qui s'intéresse à ces édifices « mégalithiques » et le lecteur y apprend beaucoup sur l'architecture et ses rapports à l'espace. La diversité des situations et des expériences présentées tout comme celle des analyses proposées, qui s'enrichissent mutuellement, disent assez le potentiel de ce sujet de recherche, loin d'être épuisé.

Christine Boujot

(Ingénieur de Recherche, DRAC Bretagne/Service régional de l'Archéologie; UMR 6566 "Civilisations atlantiques et Archéosciences ", université de Rennes 1)

Marquet, J.-C. (dir.), 2007 - L'Europe, déjà, à la fin des temps préhistoriques, catalogue d'exposition, Tours, éd. archea*, 64 p. (ISBN 978-2-912610-11-9; 8 €).

L'association en Région Centre pour l'Histoire et l'Archéologie a chargé Jean-Claude Marquet de réaliser, avec le soutien du programme Culture 2000 de l'Union européenne, une exposition consacrée au phénomène des " grandes lames de silex » en Europe. Ce projet, né en 2000 et catalysé bien évidemment par la présence en région Centre des ateliers du Grand-Pressigny, a associé la Bulgarie, l'Ukraine, la Russie

* ARCHEA : 9 rue d'Anvers, 37000 Tours; tél./fax (0)247 5165 [http:// www.archearegioncentre.org] - [archeoregioncentre@wanadoo.fr] et l'Espagne, autres pays où des industries plus ou moins comparables se sont développées. L'exposition initiale a été présentée au Muséum d'Orléans du 30 novembre 2006 au 31 janvier 2007, avant d'aller à Varna (Bulgarie) puis à Grenade (Espagne). La présentation est centré sur quatre des nombreux ensembles connus : le plateau de la Dobroudja et la nécropole de Varna au nord-est de la Roumanie, la Volhynie (région de Bodaki) en Ukraine, l'Andalousie (région de Ronda - Los Millares) en Espagne, la région pressignienne en France. 